\title{
A numerical simulation to the 3-D topography effects on the dispersions of Rayleigh Wave
}

\author{
Liu Shijie ${ }^{1, a}$, Liu Zhengping ${ }^{1, b}$ \\ ${ }^{1}$ Southwest Jiaotong University,chengdou,610031,China \\ a850869546@qq.com, 'Izhping53@sina.com
}

Keywords: Numerical simulation, Rayleigh waves, H/V ratio, Velocity dispersion

\begin{abstract}
Base on the numerical simulation, the dispersion characteristics of Rayleigh waves for 3D topographies are studied. The results show that terrains have a strong effect to the propagation of the Rayleigh waves due to the wave due to the wave conversion, reflection and diffraction. And this results strong interferences on the velocity and ellipicity dispersions of the waves. Then the convex terrain has a more complex and strong impact on the velocity and ellipcity dispersion of Rayleigh waves. The results can be hopeful for the Rayleigh survey in the mountain areas.
\end{abstract}

\section{Introduction}

Since the British scholar Lord Rayleigh in 1885 for the first time in theory found and confirmed the existence of Rayleigh surface wave in the isotropic uniform elastic medium. It is widely used in the areas of seismology, groundwater, engineering exploration, environmental geology, petroleum geology exploration and so on, because of its kinematic characteristics such as its velocity is approximately to the shear wave one and the velocity dispersion features in the inhomogeneous media. . As the dispersion feature of Rayleigh waves in the horizontal layered medium can be descried by analytic solution, so that many scholars are mainly based on the horizontal layered medium to study the propagation characteristics of Rayleigh surface waves, while the transverse non-uniformity with the convex Media research is relatively lack. In the west of China, the topography is quite complex, the mountains account for about $60 \%$ of land area. So that it is necessary to study the response characteristics of Rayleigh surface wave field to the topographies.

The study of Rayleigh surface wave in the relief terrain is mainly from the two-dimensional scattering, the main study semicircle, semi-ellipse, triangular bulge, depression and so on. The main methods of the study include finite difference method, boundary element method and so on. At present, there are relatively few studies on 3-D relief topography. Rayleigh surface wave in three-dimensional terrain research mainly using finite difference numerical simulation. Such as Lu using three-dimensional finite difference numerical simulation of Rayleigh surface wave propagation in the cracks in the law. Wang carried out finite difference numerical simulation on Rayleigh surface wave with 3-D terrain. In this paper, Commercial software abaqus is used as a tool to study the dispersion characteristics of Rayleigh surface waves under the condition of 3D topography, and the half-space model of three types of different terrain conditions including horizontal terrain is established by using abaqus. The seismic records and wavefield snapshots of different models are plotted, and the $\mathrm{H}-\mathrm{V}$ ratio is obtained. The velocity profiles are plotted according to the dispersion trend of Rayleigh wave. The influence of undulating terrain on the dispersion characteristics of Rayleigh wave is analyzed and compared.

\section{Numerical Simulation}

In order to study the effects of 3D topography on the dispersions of the Rayleigh surface wave, a model of homogeneous media with $300 \mathrm{~m} \times 300 \mathrm{~m} \times 300 \mathrm{~m}$ grid dividing was established ( figure.1 ). The hypocenter was vertical down Rayleigh wave, the main frequency was $20 \mathrm{~Hz}$, the distance from the center to the source was $140 \mathrm{~m}$. The horizontal profile of the undulating terrain is 
$80 \mathrm{~m}(4 \lambda)$ and the height of the uplift depression is $20 \mathrm{~m}(\lambda)$. The same scale horizontal terrain is also set. The P-wave velocity is $1000 \mathrm{~m} / \mathrm{s}$ and the $\mathrm{S}$-wave velocity is $400 \mathrm{~m} / \mathrm{s}$. The density is $2000 \mathrm{~kg} / \mathrm{m}$ $\wedge 3$, respectively. The excite source is set as a point vertical force in space and a ricker wavelet response in time with a $20 \mathrm{~Hz}$ main frequency. The iterative time step is $0.0005 \mathrm{~s}$. To show the topography effect clearly, we firstly simulated the horizontal topography.
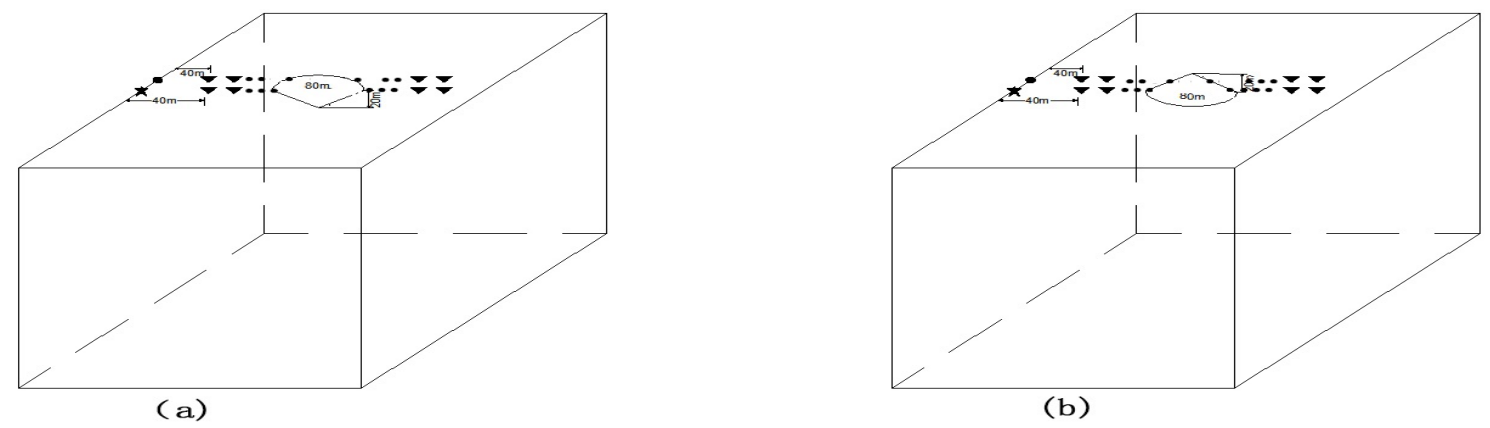

Figure. 1.The model of numerical $(\star$ and $\bullet$ is the hypocenter $\nabla$ is the Receiver point $)$
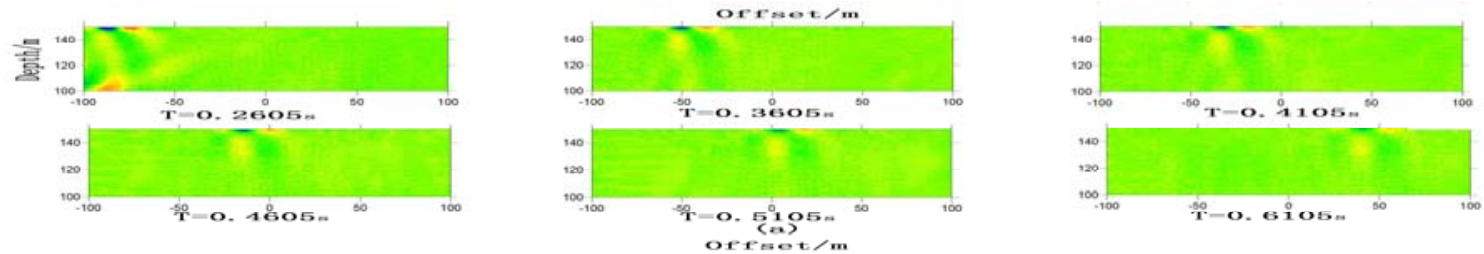

Figure. 2. The vertical components of seismic field along the centre survey line for the homogeneous half-space model with numerical simulation. (a) is the snapshot and (b) seismic record profile.
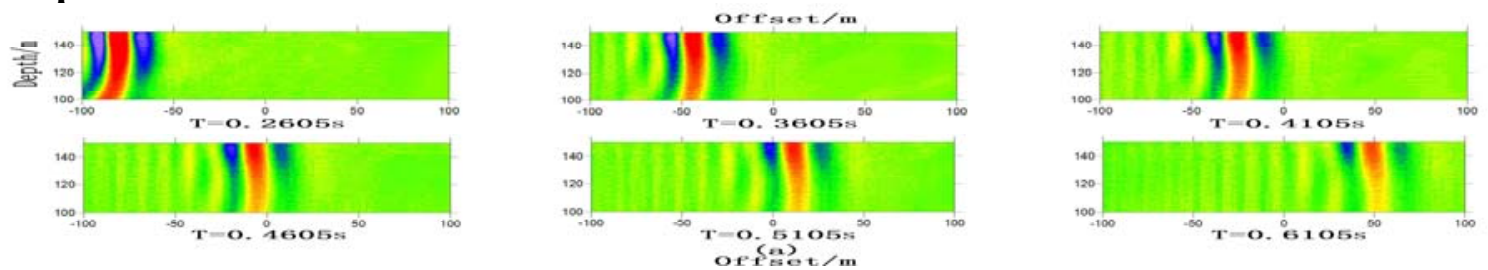

Figure. 3. The vertical components of seismic field along the centre survey line for the homogeneous half-space model with numerical simulation. (a) is the snapshot and (b) seismic record profile.

The figure 2 and 3 show the vertical and horizontal components of the snapshots and seismic record profiles of the wave field excited by the source for the horizontal topography. In the figures, the three types of wave modes can be recognized from their velocities and wavefronts. The travel velocities from fast to slow are P-wave, S- wave and Rayleigh wave, respectively. With the propagation distance increasing, the bulk wave energy near the surface attenuates and the Rayleigh wave energy increases. The propagation characteristics of the Rayleigh waves are basically the same at different times and there is no dispersion response. At the same time, the amplitude of the horizontal component of Rayleigh wave is obviously smaller than that of the vertical one. The features of the Rayleigh wave are consistent with the of the wave theory for a homogeneous half-space. 

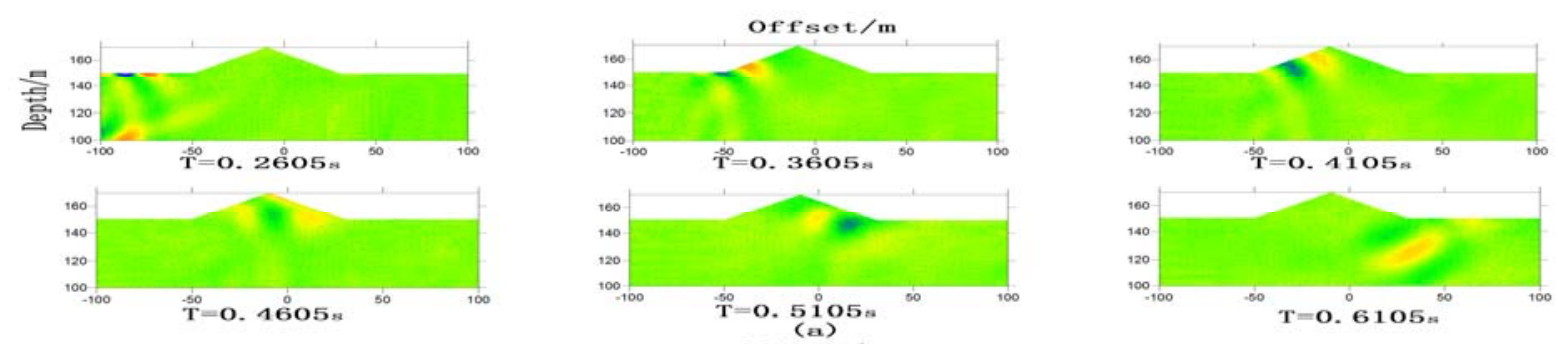

Figure. 4 .The horizantal component of seismic field along the centre survey line for the ridge model with numerical simulation. (a) is the snapshot and (b) seismic record profile .
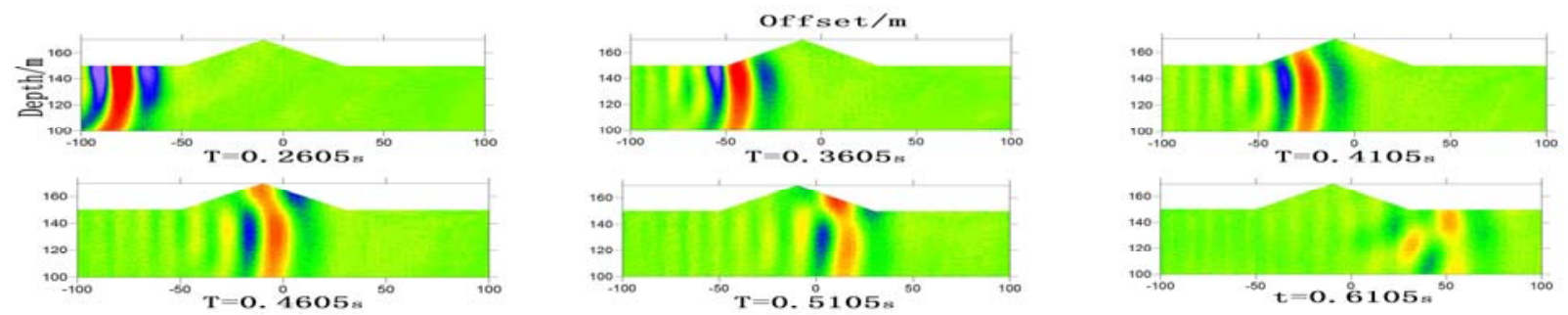

Figure. 5. The vertical component of seismic field along the centre survey line for the ridge model with numerical simulation. (a) is the snapshot and (b) seismic record profile.

Figure 4 and 5 show the simulation topography effects on the propagation of the seismic waves excited by the source for a ridge topography model. In the figures, the P-wave firstly passes through the convex terrain and has a weaker scattering. The S-wave and the Rayleigh wave arrive later. When the $\mathrm{S}$ wave and the Rayleigh wave is reaching at the top of the uplift terrain, strong transformation and scattering occur. which lead the Rayleigh wave field to become quite complex comparing with the horizontal terrain. It is also found that the amplitude of the horizontal component of the Rayleigh wave is significantly smaller than that of the vertical component.
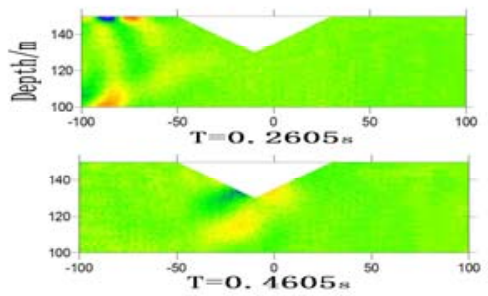
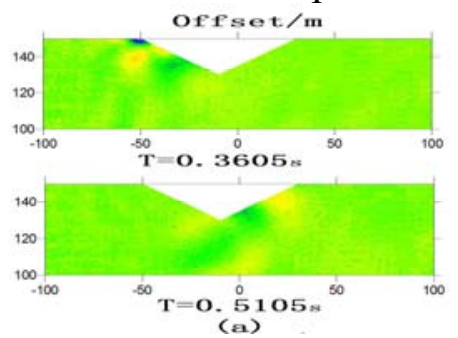
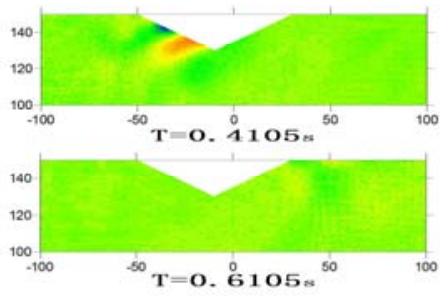

Figure. 6. The horizantal components of seismic field along the centre survey line for the valley model with numerical simulation. (a) is the snapshot and (b) seismic record profile.
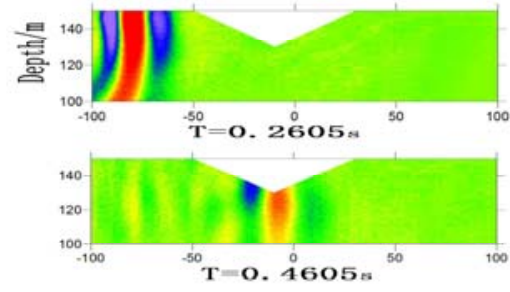
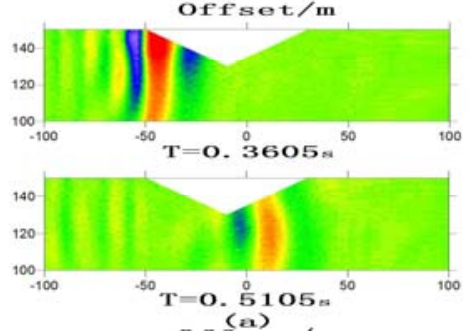
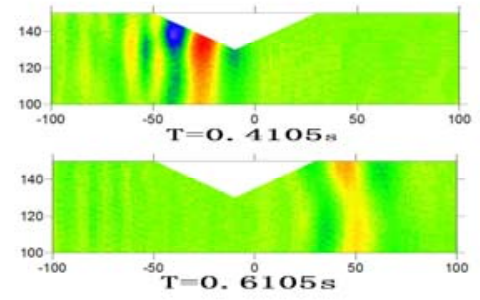

Figure. 7. The vertical component of seismic field along the centre survey line for the valley model with numerical simulation. (a) is the snapshot and (b) seismic record profile.

The numerical simulation of valley topography is shown in figure 6 and 7, respectively. Form the figures, it can be observed that the interaction between the wave field and the topography of the depressed terrain is as complex as that of the uplift terrain. The P-wave firstly arrives at the concave terrain, with the scattering energy decaying. S-wave and Rayleigh wave arrive later and then spread to the surroundings with the scattering by the terrain. 
In order to compare and analyze the impact of three kinds of terrain on the Rayleigh wave, we draw the change of the $\mathrm{H}-\mathrm{V}$ ratio of the main survey line, the lateral line of $20 \mathrm{~m}$, the survey line of the topography and the side line of $20 \mathrm{~m}$.

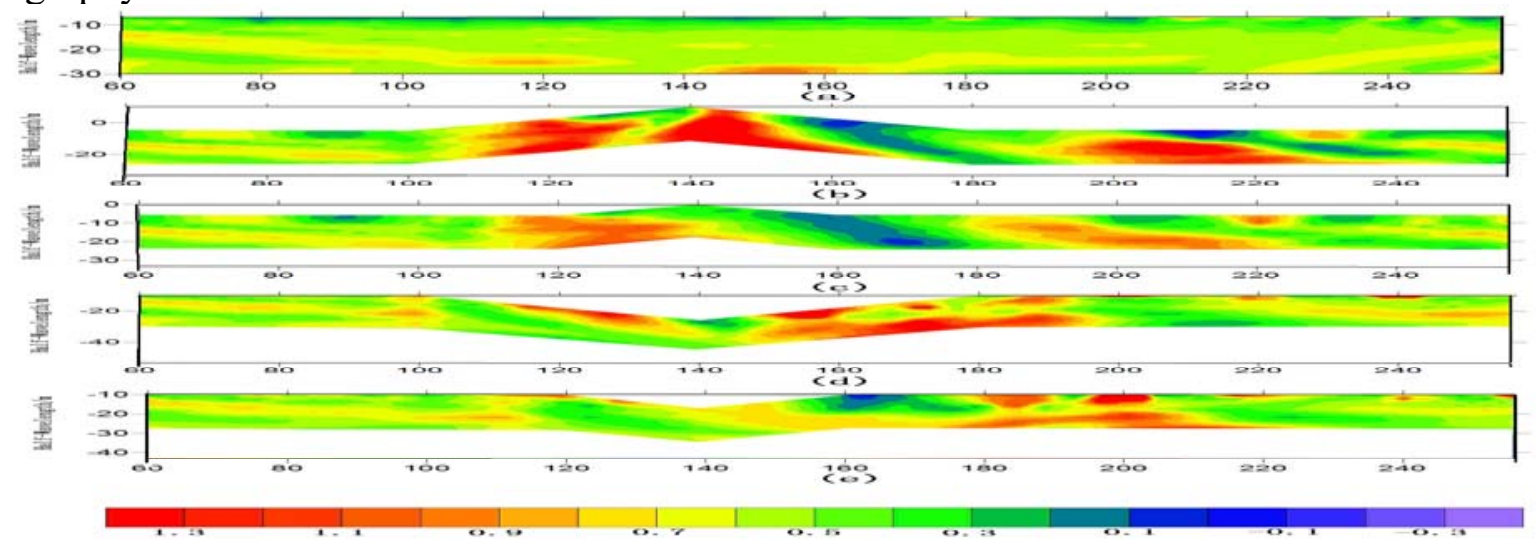

Figure. 8. The ellipticity dispersion profiles of Rayleigh waves for varying 3D topographies. (a) is the ellipticity dispersion profile of homogeneous half-space model, (b) and (c) are that of the ridge model along the centre and side survey line, respectivel, (d) and (e) are that of valley model along the centre and side survey line, respectively.

To evaluate the topography effects on the particle motion of the Rayleigh waves, we also calculate the spectrum ratio of horizontal and vertical components (simply called H/V ratio in the following discussion) for the models, which presents the ellipticity dispersion of the particle motion. It can be seen from the figure 8 that the horizontal terrain shows a relative uniform $\mathrm{H} / \mathrm{V}$ ratio distribution with few dispersion feature, though body-wave disturbance in the near-field and boundary regions resulting in some slight turbulence on the elliptic polarizability. The ellipticity of the far-field in the profile is relatively stable and distribute among the values of $0.5-0.6$, which is close the theory value 0.65. When the Rayleigh waves travel through the uplift terrain, the elliptic polarization increases significantly up to a range of 1.1-1.3 distributed near the first rise and top locations of the topography. And the decrease low to 0.1-0.3 appears after the increasing. The profile of side line shows the similar anomaly with the reduced amplitude and scale.

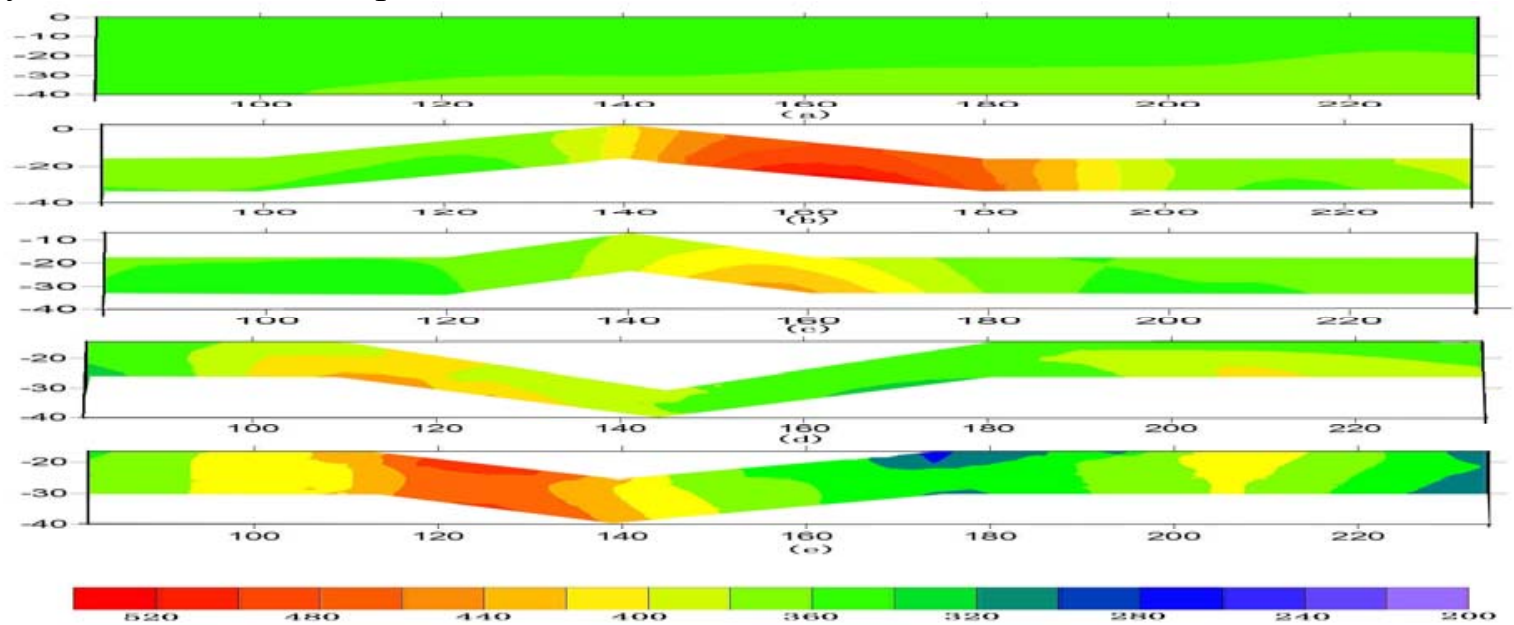

Figure. 9 . The velocity dispersion profiles of Rayleigh waves for varying 3D topographies. (a)is the velocity dispersion profile of homogeneous half-space model, (b) and (c) are that of the ridge model along the centre and side survey line, respectivel, (d) and (e) are that of valley model along the centre and side survey line, respectively.

In order to compare and analyze the impact of the 3D topographies on the velocity dispersion of Rayleigh waves, we calculates the velocity dispersion profiles of the Rayleigh waves for the centre line of the homogeneous half-space model, and the centre survey line and the side line of the ridge 
topography model and the valley model, respectively by the vertical components. The figure 8 shows that the velocity dispersion of horizontal homogeneous terrain concentrates between $360-370 \mathrm{~m} / \mathrm{s}$ being close to the theoretical surface velocity $365 \mathrm{~m} / \mathrm{s}$. Then, when the surface waves propagate through the uplift terrain along the centre line, the velocity change from a slightly reduce of $340-360 \mathrm{~m} / \mathrm{s}$, and then increase up to $500-520 \mathrm{~m} / \mathrm{s}$ after passing through the highest point of the topography. With the wave travels far from the ridge, the velocity is gradually to close the velocity of theoretical surface velocity $365 \mathrm{~m} / \mathrm{s}$. The velocity appear decrease and increase on the left and right of the topography with the wave propagating from the right to left. The similar effects can be seen from the profile of the side line with a $20 \mathrm{~m}$ distance from the centre line in the figure. It is clearly that with the ridge slope reducing, the velocity dispersion anomaly decrease rapidly. When the surface waves propagate through the valley terrain along the centre line and side line, the velocities also appear dispersion anomalies with an opposite features to that of the ridge. That is, a maximum velocity anomaly about $400-420 \mathrm{~m} / \mathrm{s}$ appear at first and then a minimum one about $300-320 \mathrm{~m} / \mathrm{s} \mathrm{dose}$ with the wave travels through the valley. The side line shows a similar velocity anomaly feature with relative weak amplitude and small area. The simulation results show that convex terrain has a more complex and strong impact on the velocity dispersion of Rayleigh waves.

\section{Conclusions}

Based on the method of numerical simulation, 3D topography effects on the dispersions of Rayleigh waves are studied. The simulating snapshots and seismic profiles show that all kinds of terrains have impacts on the Rayleigh surface wave propagation due to the wave conversion, reflection and diffraction caused by the topography. And these result strong interferences on the velocity and ellipicity dispersions of the waves. And with the decrease of the topographic relief, the effects degrease rapidly. Then the convex terrain has a more complex and strong impact on the velocity and elicit,dispersion of Rayleigh waves. The results can be a guide for the Rayleigh survey in the mountain areas.

\section{Acknowledgements}

This work was financially supported by the National Natural Science Foundation of China (Grant No.41274107).

\section{References}

[1] Aki K, Richards P G. Quantitative seismology[M]. 2002.

[2] Bard P Y. Diffracted waves and displacement field over two-dimensional elevated topographies[J]. Geophysical Journal International, 1982, 71(3): 731-760.

[3] Hong Z, Xiao-Fei C. A study on the effect of depressed topography on Rayleigh surface wave[J]. CHINESE JOURNAL OF GEOPHYSICS-CHINESE EDITION, 2007, 50(4): 1182-1189.

[4] Tsaur D H. Scattering and focusing of SH waves by a lower semielliptic convex topography[J]. Bulletin of the Seismological Society of America, 2011, 101(5): 2212-2219.

[5] Wang L, Luo Y, Xu Y. Numerical investigation of Rayleigh-wave propagation on topography surface[J]. Journal of Applied Geophysics, 2012, 86: 88-97.

[6] Wei C, Yixian X, Yu Z. Study of Transmission Characteristics of Rayleigh Waves on an Elastic Wedge[J]. Chinese Journal of Engineering Geophysics, 2009, 2: 002.

[7] $\mathrm{Yu} \mathrm{W,} \mathrm{Liu} \mathrm{Z.} \mathrm{A} \mathrm{numerical} \mathrm{study} \mathrm{of} \mathrm{the} \mathrm{Rayleigh} \mathrm{wave} \mathrm{particle} \mathrm{motions} \mathrm{excited} \mathrm{by} \mathrm{a} \mathrm{point} \mathrm{source}$ and Poisson's ratio for lateral inhomogeneous half-spaces[J]. Journal of Applied Geophysics, 2015, 123: 242-255. 